\title{
Der Sauerstoffhaushalt von Fliessgewässern: Kritische Prüfung eines mathematischen Sauerstoffmodells anhand der Identifikation seiner Parameter in Flüssen und künstlichen Rinnen
}

\author{
Von P.E. Erni und J. Ruchti \\ Eidgenössische Anstalt für Wasserversorgung, Abwasserreinigung und Gewässerschutz (EAWAG), \\ CH-8600 Dübendorf
}

Manuskript eingegangen am 9.Januar 1978

ABSTRACT

Results obtained by a previously published cross-correlation identification technique applied to the modified Streeter-Phelps river model suggest that the oxygen exchange rate, the oxygen invasion rate, an apparent photosynthesis rate and the respiration rate can be identified using oxygen measurements from a single point of observation. This hypothesis is examined here using the alternative method of differential approximation and the gradient method. It is found that the simple solely time dependent model is inadequate for the description of the dissolved oxygen concentration in a river and that consequently its parameters can not be identified using the measurements from a single point only.

\section{Einleitung}

Schon früh (Streeter und Phelps [1]) wurde erkannt, dass die Selbstreinigung eines Flusses wesentlich von der Sauerstoffaustauschrate zwischen Fluss und Atmosphäre abhängt. Dieser Parameter kann sowohl direkt als auch indirekt über ein mathematisches Modell bestimmt werden. Grundlegende Arbeiten über die direkte Parameterbestimmung mit radioaktiven Tracern stammen hauptsächlich von Tsivoglou et al. [2] und Longtin [3]. O'Connor [4], Dobbins [5] und Churchill et al. [6] befassten sich dagegen neben Streeter und Phelps mit der indirekten modellmässigen Bestimmung der Sauerstoffaustauschrate.

Neben der Sauerstoffaustauschrate sind auch die Photosynthese- und die Atmungsrate der sich im Fluss befindenden Pflanzen und Mikroorganismen in hohem Masse für den Sauerstoffhaushalt des Flusses verantwortlich. Aufgrund dieser Tatsache versuchten Koivo und Koivo [7] aus der Sauerstoffkonzentration mit Hilfe der Estimationstheorie auf die biologischen Grössen (biologischer Sauerstoffbedarf oder totaler organischer Kohlenstoff) zu schliessen. In einer neueren Arbeit von Schurr und Ruchti [8] wurden die Modellparameter mit Hilfe von Kreuzkorrelationen identifiziert. Da die Identifikation mit Hilfe von Sauerstoffmessungen nur 
einer Elektrode erfolgen sollte, musste im Modell die Abhängigkeit der Sauerstoffkonzentration in Funktion des Ortes vernachlässigt werden. Die Autoren legten bei der Identifikation grossen Wert darauf, den Einfluss von Wolken. Nebel und anderen zufälligen Störungen der Sonneneinstrahlung auf die Photosynthese zu berücksichtigen. Das führte dazu. dass bei der Identifikation nicht die in der Modellgleichung vorkommende Photosyntheserate erhalten wird, sondern das Produkt aus Photosyntheserate, künstlich eingeführtem Opazitätsfaktor und mittlerer Lichtintensität. Im weiteren wurde in dieser Arbeit gezeigt, dass das Modell nur dann vernünftige Resultate liefert, wenn die Sauerstoffkonzentration nicht in der Nähe von Wasserfällen, Stromschnellen usw. gemessen wird.

Ein Nachteil der Methode von Schurr und Ruchti besteht darin, dass das Modell nicht verifiziert werden kann, weil die Methode die im Modell vorkommende Photosyntheserate nicht identifiziert. Ausserdem erscheint uns die Parameteridentifikation mit Hilfe von Kreuzkorrelationen relativ umständlich und rechenintensiv. Mit der Differentialapproximation und der Gradientenmethode stehen zwei Algorithmen zur Verfügung, die mathematisch einfach sind und rasch arbeiten. Die erhaltenen Resultate werden zusammengestellt und diskutiert.

\section{Das Modell}

Das von Schurr und Ruchti aufgestellte Modell gilt unter den folgenden Voraussetzungen:

- Die Änderung der Sauerstoffkonzentration in der Fliessrichtung des Flusses wird vernachlässigt.

- Die geometrische Ausdehnung des Flusses wird nicht berücksichtigt.

- Die Lichtintensität ist nur eine Funktion der Zeit.

- Die Raten für den Sauerstoffeintrag, die Photosynthese und die Atmung sind nicht ortsabhängig.

- Die Sauerstoffkonzentration ändert sich nicht in vertikaler Richtung.

- Die Photosynthese ist nur abhängig vom einfallenden Licht (Nährstoffe sind im Überschuss vorhanden).

Unter diesen Annahmen lauten die Modellgleichungen:

$$
\mathrm{O}(\mathrm{t})=-\widehat{\mathrm{k}} \cdot \mathrm{O}(\mathrm{t})+\overrightarrow{\mathrm{k}} \mathrm{O}_{\mathrm{A}}+\alpha \cdot \mathrm{I}(\mathrm{t})-\beta
$$

und

$$
\frac{\overrightarrow{\mathrm{k}} \mathrm{O}_{\mathrm{A}}}{\overrightarrow{\mathrm{k}}}=\mathrm{O}_{\mathrm{eq}}
$$

mit

$\overline{\mathrm{k}}=$ Rate der Sauerstoffabgabe an die Atmosphäre

$\overrightarrow{\mathrm{k}}=$ Sauerstoffeintragsrate

$a=$ Photosyntheserate

$\beta=$ Atmungsrate 
$\mathrm{O}_{\mathrm{A}}=$ Sauerstoffkonzentration in der Atmosphäre

$\mathrm{O}_{\mathrm{eq}}=$ Sauerstoffkonzentration bei Sättigung

$O(t)=$ Sauerstoffkonzentration im Fluss

$I(t)=$ Lichtintensität $(0 \leqslant I(t) \leqslant 1)$

Für die Anwendung der Identifikationsalgorithmen ist es notwendig, die Modellgleichungen (1) und (2) als Differenzengleichung zu formulieren:

$$
\mathrm{O}(\mathrm{k}+\mathrm{l})=\mathrm{a} \cdot \mathrm{O}(\mathrm{k})+\mathrm{b} \cdot \mathrm{I}(\mathrm{k})+\mathrm{c}
$$

mit

$$
\begin{aligned}
& \mathrm{a}=\mathrm{e}^{-\overline{\mathrm{k}} \cdot \mathrm{T}_{\mathrm{s}}} \\
& \mathrm{b}=-\frac{a}{\overline{\mathrm{k}}} \cdot\left(\mathrm{e}^{-\mathrm{k} \cdot \mathrm{T}_{\mathrm{s}}}-1\right), \\
& \mathrm{c}=\frac{\beta-\overrightarrow{\mathrm{k}} \mathrm{O}_{\mathrm{A}}}{\overline{\mathrm{k}}} \cdot\left(\mathrm{e}^{-\hat{k} \cdot T_{S}}-1\right) .
\end{aligned}
$$

$\mathrm{T}_{\mathrm{S}}=$ Abtastperiode (Zeit zwischen zwei Messpunkten).

Gleichung (3) wird im Anhang hergeleitet.

\section{Identifikationsmethoden}

Die verwendeten Identifikationsmethoden, die Differentialapproximation und die Gradientenmethode, sind mathematisch einfach und problemlos in der Anwendung. Sie bedingen jedoch, dass das mathematische Modell linear in den Parametern ist. Linear in den Parametern bedeutet, dass der Vektor â mit Hilfe der Messgrössen $\mathrm{z}, \mathbf{y}$ aus der Gleichung

$$
\mathrm{z}=\mathbf{a}^{\prime} \cdot \mathbf{y}
$$

berechnet werden kann. Für das System

$$
\mathrm{O}(\mathrm{k}+\mathrm{l})=\mathrm{a} \cdot \mathrm{O}(\mathrm{k})+\mathrm{b} \cdot \mathrm{I}(\mathrm{k})+\mathrm{c}
$$

ergeben sich für $\mathbf{z}, \mathbf{a}^{\prime}$ und $\mathbf{y}$ :

$$
\begin{aligned}
& z(k)=O(k+1), \\
& \mathbf{a}^{\prime}=[a b c], \\
& y=\left[\begin{array}{l}
O(k) \\
I(k) \\
1
\end{array}\right] .
\end{aligned}
$$




\subsection{Differentialapproximation}

Diese Methode [9] liefert für die Gesamtheit aller Werte von $O(k)$ und I (k) einen Wert für jeden zu identifizierenden Parameter. Schwankungen der einzelnen Parameter in Funktion der Zeit werden ausgemittelt. Die Differentialapproximation eignet sich daher nur zur nachträglichen Identifikation der Parameter.

Das zu lösende Gleichungssystem lautet wie folgt:

$$
\begin{aligned}
& a \sum_{k=1}^{N-1} O^{2}(k)+b \sum_{k=1}^{N-1} O(k) \cdot I(k)+c \sum_{k=1}^{N-1} O(k)=\sum_{k=1}^{N-1} O(k) \cdot O(k+1) \\
& a \sum_{k=1}^{N-1} O(k) \cdot I(k)+b \sum_{k=1}^{N-1} I^{2}(k)+c \sum_{k=1}^{N-1} I(k)=\sum_{k=1}^{N-1} I(k) \cdot O(k+1) \\
& a \sum_{k=1}^{N-1} O(k)+b \sum_{k=1}^{N-1} I(k) \quad+c \sum_{k=1}^{N-1} 1=\sum_{k=1}^{N-1} O(k+1)
\end{aligned}
$$

mit $\mathrm{N}=$ Anzahl Messwerte pro Meßserie

$a, b, c=z u$ identifizierende Parameter

\subsection{Die Gradientenmethode}

Diese Methode [10] liefert für je zwei aufeinanderfolgende Werte von $O(k)$ und $\mathrm{I}(\mathrm{k})$ je einen Wert für jeden zu identifizierenden Parameter. Die Gradientenmethode wird deshalb dann mit Vorteil verwendet, wenn die Parameter direkt (on line) ermittelt werden sollen. Der Algorithmus lautet wie folgt:

$$
\hat{\mathbf{a}}(\mathrm{k}+1)=\hat{\mathbf{a}}(\mathrm{k})+\frac{\mathrm{E}}{\mathbf{y}^{\prime}(\mathrm{k}) \cdot \mathbf{y}(\mathrm{k})} \cdot\left(\mathrm{z}(\mathrm{k})-\hat{\mathbf{a}}^{\prime}(\mathrm{k}) \cdot \mathbf{y}(\mathrm{k})\right) \cdot \mathbf{y}(\mathrm{k})
$$

mit

$$
\begin{aligned}
& \hat{\mathbf{a}}(\mathrm{k}+1)=\left[\begin{array}{l}
\mathrm{a}(\mathrm{k}+1) \\
\mathrm{b}(\mathrm{k}+1) \\
\mathrm{c}(\mathrm{k}+1)
\end{array}\right] \quad \begin{array}{l}
\text { zu identifizierender Parametervektor } \\
\text { nach dem } \mathrm{k}+1 . \text { Schritt }
\end{array} \\
& \hat{\mathbf{a}}(\mathrm{k})=\left[\begin{array}{l}
\mathrm{a}(\mathrm{k}) \\
\mathrm{b}(\mathrm{k}) \\
\mathrm{c}(\mathrm{k})
\end{array}\right] \quad \begin{array}{l}
\text { zu identifizierender Parametervektor } \\
\text { nach dem k-ten Schritt }
\end{array} \\
& \mathbf{y}(\mathrm{k})=\left[\begin{array}{l}
\mathrm{O}(\mathrm{k}) \\
\mathrm{I}(\mathrm{k}) \\
1
\end{array}\right] \\
& \mathrm{z}(\mathrm{k})=\mathrm{O}(\mathrm{k}+1) \\
& \mathrm{E}=\text { Einheitsmatrix }
\end{aligned}
$$




\section{Messdaten}

Die Modellparameter wurden an Daten identifiziert, die an der Aare bei Bern (1.3.74-31.3.74) und Brugg (1.1.72-31.1.72) erhoben worden sind und uns vom Eidg. Amt für Wasserwirtschaft zur Verfügung gestellt wurden. Diese beiden Datensätze, bei denen die gemessene Sauerstoffkonzentration extrem verschieden ist, wurden schon von Schurr und Ruchti zur Parameteridentifikation herbeigezogen. Bei den Daten von Bern ist der Einfluss der Lichtintensität auf die Sauerstoffkonzentration deutlich zu erkennen (Abb. 1), während dies bei den Daten von Brugg (Abb. 2) nicht der Fall ist oder zu sein scheint [8].

Ausserdem wurden die Modellparameter noch an Daten identifiziert, welche an den künstlichen Rinnen der Versuchsanlage der EAWAG in der Tüffenwies gemessen wurden. Diese Daten wurden von Wetzel [11] in der Zeit vom 19.6 .74 bis 21.6 .74 erhoben.

Damit die Parameter identifiziert werden können, müssen die folgenden Daten bekannt sein:

- Sauerstoffkonzentration,

- Temperatur,

- Lichtintensität,

- Geographische Daten der Messorte.

\subsection{Sauerstoffmessung (Abb. 1 und 2)}

Die Sauerstoffdaten der Aare, welche mit einer amperometrischen Sauerstoffelektrode gemessen wurden, sind uns in Form von Meßstreifen vom Eidg. Amt für Wasserwirtschaft zur Verfügung gestellt worden. Die Kurven wurden digitalisiert und so aufbereitet, dass der zeitliche Abstand von zwei Messpunkten 20 Minuten beträgt. Für eine Meßserie von einem Monat zu 31 Tagen erhält man somit 2233 Messpunkte.

Die Sauerstoffdaten der Rinne wurden ebenfalls mit einer amperometrischen Sauerstoffelektrode gemessen. Die Daten wurden so aufbereitet, dass die Abstand

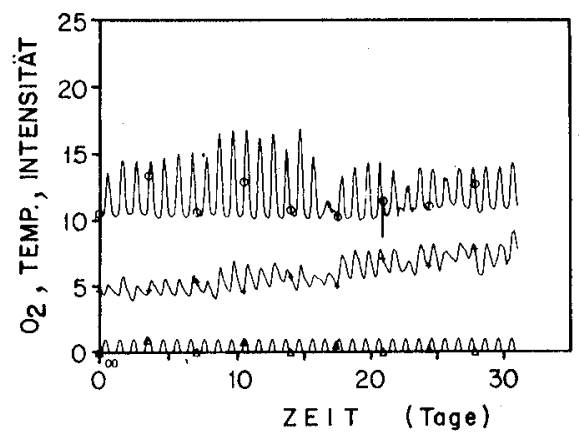

Abb. 1. Messdaten Aare bei Bern $(O=$ Sauerstoffkonzentration, $+=$ Temperatur, $\Delta=$ Lichtintensität $)$. Figure 1. Raw data in the Aare river at Bern $(\mathrm{O}=$ dissolved oxygen, $+=$ temperature, $\Delta=$ light intensity). 


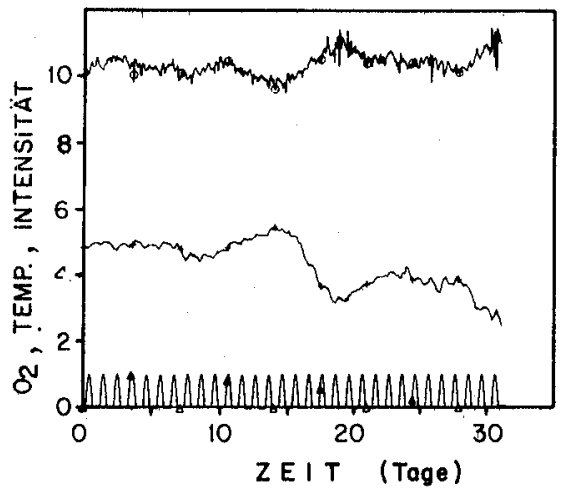

Abb.2. Messdaten Aare bei Brugg $(O=$ Sauerstoffkonzentration, $+=$ Temperatur, $\Delta=$ Lichtintensität $)$.

Figure 2. Raw data in the Aare river at Brugg $(O=$ dissolved oxygen, $+=$ temperature, $\Delta=$ light intensity).

von zwei Messpunkten 10 Minuten beträgt, was für eine Meßserie von 3 Tagen 433 Messpunkte ergibt.

\subsection{Temperaturmessungen (Abb. 1 und 2)}

Die Temperaturmesswerte sind notwendig, da die in Gleichung (2) vorkommende Sauerstoffkonzentration bei Sättigung $\mathrm{O}_{\mathrm{eq}}$ temperaturabhängig ist. Die Temperaturmesswerte sind auf die gleichen Meßstreifen aufgezeichnet worden, wie die Messwerte der Sauerstoffkonzentration. Die Messwerte wurden für die AareDaten in 2233 Messpunkte und für die Rinnendaten in 433 Messpunkte digitalisiert.

\subsection{Lichtintensität}

Die Lichtintensität (Steuergrösse) wurde für die Aare-Daten nicht gemessen. Sie musste daher mit der folgenden Funktion simuliert werden:

$$
\left.I(t)=\left\{\begin{array}{c}
\cos (\pi / 12 \cdot t) \\
0
\end{array}\right\} \begin{array}{c}
T_{m}-6 \leqslant t \leqslant T_{m}+6 \\
\left\{\begin{array}{l}
T_{m}-12 \leqslant t<T_{m}-6 \\
T_{m}+6<t<T_{m}+12
\end{array}\right\}
\end{array}\right\}
$$

mit $T_{m}=$ geographische Mittagszeit.

In der Tüffenwies wurde die Lichtintensität mit Hilfe eines Kipp- und ZonenSolarimeters (Messbereich 300-3000 nm) gemessen und anschliessend in 433 Messpunkte digitalisiert. 


\subsection{Geographische Daten der Messorte}

Die Sauerstoffkonzentration bei Sättigung $\mathrm{O}_{\mathrm{eq}}$ hängt neben der Temperatur noch von der Höhe über Meer ab. Die Meßstellen bei Bern, Brugg und in der Tüffenwies liegen 505 bzw. 330 bzw. 466 Meter über Meer.

Die geographische Mittagszeit $T_{m}$, welche in der Gleichung (13) vorkommt, hängt von der geographischen Länge des Messortes ab. Der geographische Mittag ist in Bern um 12.50, in Brugg um 12.45 und in der Tüffenwies um 12.43 Uhr.

\section{Resultate der Identifikation}

Die im Abschnitt 3 aufgeführten Identifikationsalgorithmen wurden sowohl auf die Daten von Bern und Brugg als auch auf die Rinnendaten angewendet.

Die numerischen Resultate für alle Meßstellen sind in der Tabelle zusammengefasst. Bei der Differentialapproximation ist das Fehlerquadratintegral $\mathrm{Z}$ relativ gross, da nur ein Satz von Parametern erhalten wird. Eine signifikante Reduktion des Fehlerquadratintegrals konnte bei den Daten von Bern erreicht werden durch eine (durch Identifikation erhaltene) Vorverschiebung der Lichtintensitätsfunktion um 0,8 Stunden. Mit der Gradientenmethode erhält man, wie nicht anders zu erwarten war, ein sehr kleines Fehlerquadratintegral und somit eine äusserst gute Übereinstimmung der berechneten mit der gemessenen Sauerstoffkonzentration.

Abb. 3 zeigt das Resultat der Simulation mit den aus der Differentialapproximation erhaltenen Parameter für die Daten von Bern. Dabei wurde die oben erwähnte Vorverschiebung der Lichtintensitätsfunktion bereits berücksichtigt. Die berechnete Sauerstoffkonzentration folgt der gemessenen ziemlich gut, jedoch bleibt die Ampli-

Numerische Resultate der Identifikationen.

Numerical results of the identifications.

\begin{tabular}{|c|c|c|c|c|c|c|}
\hline & \multicolumn{2}{|c|}{ Bern } & \multicolumn{2}{|c|}{ Brugg } & \multicolumn{2}{|c|}{ Rinne } \\
\hline & $\begin{array}{l}\text { Diffe- } \\
\text { rential- } \\
\text { approxi- } \\
\text { mation }\end{array}$ & $\begin{array}{l}\text { Gradienten- } \\
\text { methode }\end{array}$ & $\begin{array}{l}\text { Diffe- } \\
\text { rential- } \\
\text { approxi- } \\
\text { mation }\end{array}$ & $\begin{array}{l}\text { Gradienten- } \\
\text { methode }\end{array}$ & $\begin{array}{l}\text { Diffe- } \\
\text { rential- } \\
\text { approxi- } \\
\text { mation }\end{array}$ & $\begin{array}{l}\text { Gradienten- } \\
\text { methode }\end{array}$ \\
\hline $\mathrm{k}_{\min }$ & - & $-0,311$ & - & $-0,189$ & - & $-0,281$ \\
\hline $\mathrm{k}_{\text {mittel }}$ & 0,244 & 0,246 & 0,053 & 0,053 & 0,042 & 0,046 \\
\hline $\mathrm{k}_{\max }$ & - & 0,613 & - & 0,215 & - & 0,337 \\
\hline $\overrightarrow{\mathrm{k}} \mathrm{O}_{\mathrm{A}_{\text {min }}}$ & - & $-3,515$ & - & $-2,453$ & - & $-3,020$ \\
\hline $\overrightarrow{\mathrm{k}} \mathrm{O}_{\mathrm{A}_{\text {mittel }}}$ & 2,837 & 2,874 & 0,656 & 0,668 & 0,456 & 0,496 \\
\hline $\overrightarrow{\mathbf{k}} \mathrm{O}_{\mathrm{A}_{\max }}$ & - & 6,896 & - & 2,796 & - & 3,565 \\
\hline$a_{\min } \max$ & - & 1,266 & - & 0,0050 & - & 0,069 \\
\hline$a_{\text {mittel }}$ & 1,411 & 1,391 & 0,0047 & 0,0067 & 0,112 & 0,101 \\
\hline$a_{\max }$ & - & 1,473 & - & 0,026 & - & 0,135 \\
\hline$\beta_{\min }$ & - & $-5,837$ & - & $-3,015$ & - & $-3,328$ \\
\hline$\beta_{\text {mittel }}$ & 0,403 & 0,407 & 0,112 & 0,118 & 0,188 & 0,202 \\
\hline$\beta_{\max }$ & - & 4,304 & - & 2,238 & - & 3,280 \\
\hline Fehlerquadratintegral Z & 1594 & 28,3 & 209 & 7,6 & 596 & 3,9 \\
\hline Zeitverschiebung $T_{z}[h]$ & 0,8 & - & - & - & - & - \\
\hline Fehlerquadratintegral $Z_{T_{2}}$ & 1347 & - & - & - & - & - \\
\hline
\end{tabular}




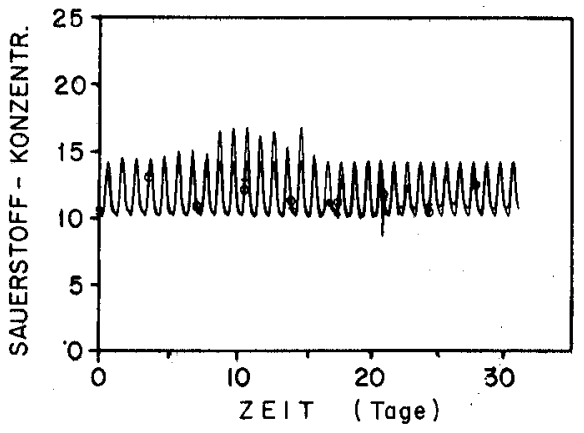

Abb.3. Berechnete Sauerstoffkonzentration für die Daten von Bern. Die Parameter wurden mit der Differentialapproximation erhalten $(O=$ berechnete Sauerstoffkonzentration, $+=$ gemessene Sauerstoffkonzentration).

Figure 3. Calculated concentration of dissolved oxygen for the data of Bern. The parameters were obtained with the method of differential approximation $(O=$ calculated concentration of dissolved oxygen, $+=$ measured concentration of dissolved oxygen).
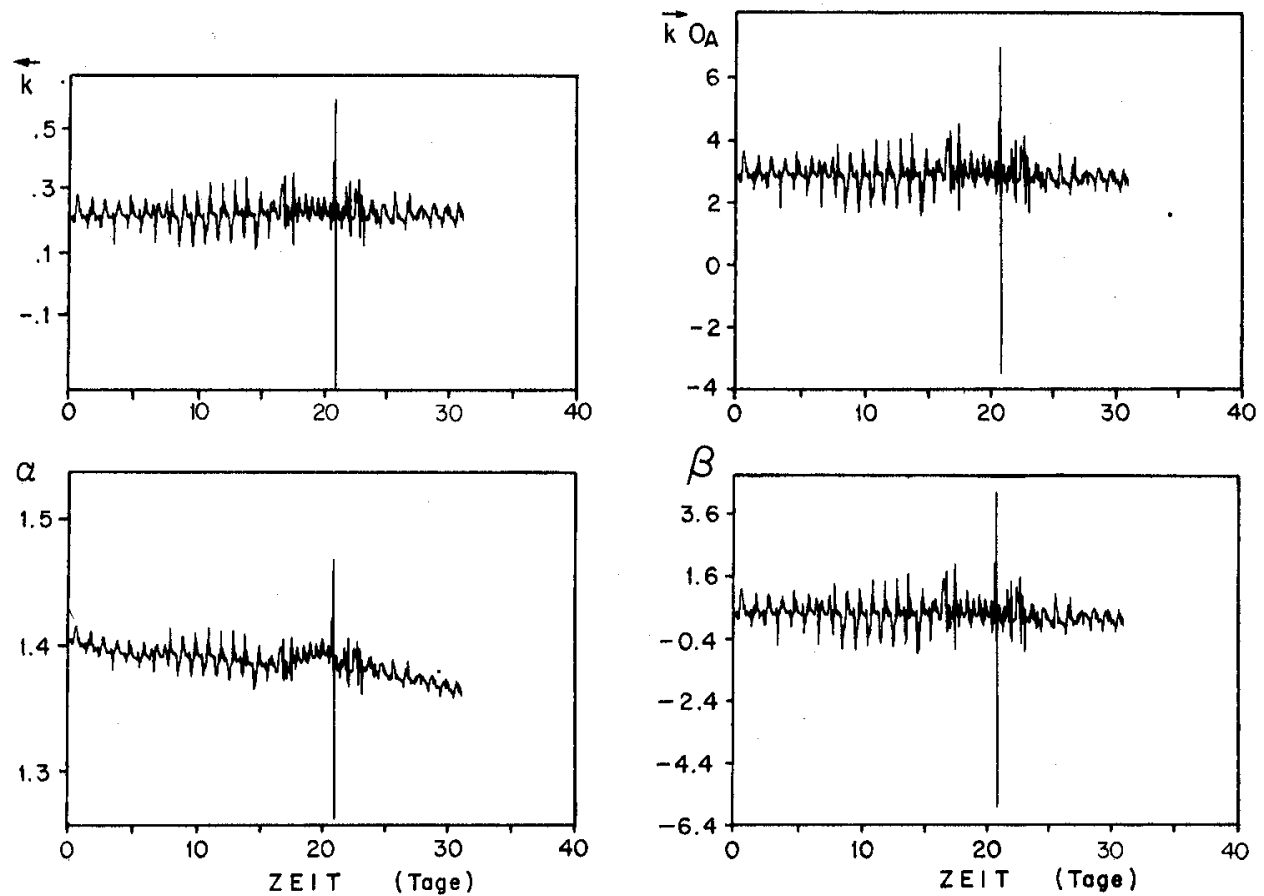

Abb.4. Mit der Gradientenmethode erhaltene Werte der Parameter $\overrightarrow{\mathrm{k}}, \overrightarrow{\mathrm{k}} \mathrm{O}_{\mathrm{A}}, \alpha$ und $\beta$ für die Daten von Bern.

Figure 4. Values of the parameters $\overrightarrow{\mathrm{k}}, \overrightarrow{\mathrm{k}} \mathrm{O}_{\mathrm{A}}, \alpha$ and $\beta$ obtained with the gradient method for the data of Bern. 
tude der täglichen Schwankungen bei der berechneten Sauerstoffkonzentration konstant, so dass sie nicht allen Schwankungen der gemessenen Sauerstoffkonzentration folgen kann.

Abb. 4 zeigt den Verlauf der mit der Gradientenmethode identifizierten Parameter für die Daten von Bern. Abgesehen von den überlagerten Spitzen bleiben die Parameter $\overleftarrow{\mathrm{k}}, \overrightarrow{\mathrm{k}} \mathrm{O}_{\mathrm{A}}$ und $\beta$ ziemlich konstant. Der Parameter $a$ nimmt in Funktion der Zeit leicht ab. Die Spitzen werden hauptsächlich dadurch hervorgerufen, dass die synthetische Lichtintensitätsfunktion nicht mit der wirklichen Sonneneinstrahlung übereinstimmt. Die Abnahme von $a$ kann damit erklärt werden, dass die Sonneneinstrahlung wegen der im März rasch länger werdenden Tage zunimmt, oder $a$ hängt umgekehrt proportional von der Temperatur ab. Die extrem hohe Spitze bei $t=21$ Tagen rührt von einem Fehler in der Sauerstoffmessung her.

Wie aufgrund der Arbeit von Schurr und Ruchti zu erwarten war, werden für die Daten von Brugg zum Teil unerklärbare Resultate erhalten. Bei der Simulation mit den aus der Differentialapproximation erhaltenen Parameter erhält man für die Sauerstoffkonzentration praktisch eine Gerade, welche den Schwankungen der gemessenen Sauerstoffkonzentration überhaupt nicht folgt. Auch die mit der Gradientenmethode erhaltenen Parameter weisen in Funktion der Zeit unregelmässig verteilt hohe Spitzen auf, welche nicht interpretiert werden können.

Auch für die Rinnendaten ergeben die Identifikationsalgorithmen unbrauchbare Resultate. Bei der Simulation mit den aus der Differentialapproximation erhaltenen Parametern erhält man eine sehr schlechte Übereinstimmung zwischen der gemessenen und der berechneten Sauerstoffkonzentration, obwohl die Sauerstoffkonzentration stark von der Lichtintensität abhängt. Die mit der Gradientenmethode erhaltenen Parameter weisen wiederum nicht interpretierbare, unregelmässig ver-. teilte Spitzen auf.

\section{Diskussion}

In dieser Arbeit wird das gleiche Modell wie in der Arbeit von Schurr und Ruchti verwendet. Es seien zuerst die Resultate der Differentialapproximation mit jener Arbeit verglichen.

- Die von Schurr und Ruchti mit der Kreuzkorrelation erhaltenen Parameter sind ungefähr um den Faktor 2 grösser als die mit der Differentialapproximation erhaltenen Parameter (Schurr/Ruchti: $\overleftarrow{\mathrm{k}}=0,386, \overrightarrow{\mathrm{k}} \mathrm{O}_{\mathrm{A}}=4,6, \beta=0,6$ für Bern und $\overleftrightarrow{\mathrm{k}}=0,12, \overrightarrow{\mathrm{k}} \mathrm{O}_{\mathrm{A}}=1,48, \beta=0,25$ für Brugg).

- Die numerischen Werte der Photosyntheseraten können nicht miteinander verglichen werden, da in der Arbeit von Schurr und Ruchti in diesem Parameter noch die Abhängigkeit der Lichtintensität vom Wetter enthalten ist.

- Da aus der Photosyntheserate in der Arbeit von Schurr und Ruchti das in Gleichung (1) vorkommende $a$ nicht extrahiert werden kann, ist es auch nicht möglich, eine Simulation durchzuführen. Erst ein Vergleich der Fehlerquadratintegrale würde zeigen, ob die Identifikation mit Kreuzkorrelationen oder die Differentialapproximation bessere Werte für die Parameter liefert.

- Schurr und Ruchti wählten für die synthetische Lichtintensität die folgende periodische Funktion: 
$\left.I(t)=\left\{\begin{array}{c}0,5 \cdot[1+\cos (\pi / 6 \cdot t)] \\ 0\end{array}\right\} \begin{array}{l}T_{m}-6 \leqslant t \leqslant T_{m}+6 \\ T_{m}-12 \leqslant t<T_{m}-6 \\ T_{m}+6<t<T_{m}+12\end{array}\right\}$

Die Identifikation mit der Differentialapproximation und der Gradientenmethode mit anschliessender Simulation ergab für die obige Lichtintensitätsfunktion (14) stets ein grösseres Fehlerquadratintegral als für die abgeschnittene Sinusfunktion (13). Ėfektiv gemessene Lichtintensitätsfunktionen haben - an einem wolkenlosen Tag - in der Tat eher die Form einer abgeschnittenen Sinusfunktion.

Die in dieser Arbeit verwendeten Identifikationsalgorithmen erlauben es, den Fehler des Modells abzuschätzen:

- Aus den erhaltenen Resultaten geht wie bei der Arbeit von Schurr und Ruchti hervor, dass das Modell nur für die Daten von Bern anscheinend plausible Resultate liefert. Der Grund für das offensichtliche Versagen des Modells für die Daten von Brugg liegt darin, dass die Meßstation kurz nach einem Stauwehr liegt. Das hat zur Folge, dass die Sauerstoffkonzentration nur noch in sehr geringem Masse von der Lichtintensität abhängt, was auch in einem sehr kleinen numerischen Wert von $a \quad(\alpha=0,0047$ bei Brugg, im Gegensatz zu $\alpha=1,411$ bei Bern) zum Ausdruck kommt. Der vermutete Sauerstoffeintrag durch das Stauwehr verletzt die Modellannahme der Ortsunabhängigkeit der Sauerstoffkonzentration.

- Um den Einfluss der Ortsabhängigkeit des Sauerstoffs auf die Identifikation zu untersuchen, wurden Messdaten an einer künstlichen Rinne untersucht, deren Sauerstoffkonzentration im Längsprofil dauernd stark anstieg (Abb. 5). Die Resultate (Tabelle) bestätigen diese Vermutung. Die Ungenauigkeit des Modells ist in diesem Fall sogar so gross, dass die einfache Gleichung

$O(t)=a^{*} \cdot I(t)+b^{*}$

mit $\mathrm{a}^{*}=4,06$ und $\mathrm{b}^{*}=6,12$ bei der Simulation ein kleineres Fehlerquadratinte$\operatorname{gral}(\mathrm{Z}=79,0)$ liefert.

- Für die Daten von Bern wird ein wesentlich besseres Fehlerquadratintegral erhalten, wenn die Lichtintensitätsfunktion I(t) um 0,8 Stunden vorverschoben wird. Diese Verschiebung der Lichtintensitätsfunktion entspricht folgender Gleichung:

$\mathrm{O}(\mathrm{t})=-\overleftarrow{\mathrm{k}} \cdot \mathrm{O}(\mathrm{t})+\overrightarrow{\mathrm{k}} \mathrm{O}_{\mathrm{A}}+\alpha \mathrm{I}(\mathrm{t}+\delta)-\beta$.

Der Wert von $\delta=-0,8$ lässt sich physikalisch nicht erklären. Die Tatsache, dass die Identifikation mit $\delta \neq 0$ bessere Resultate liefert, ist ein klarer Hinweis, dass das Modell [Gleichungen (1) und (2)] nicht zutrifft. Das erhaltene $\delta \neq 0$ lässt sich aus der Arbeitsweise der Identifikationsmethode wie folgt erklären:

Mit der Vorverschiebung von $I(t)$ wird das Nacheilen der berechneten Sauerstoffkonzentration $O(t)$ gegenüber der gemessenen Sauerstoffkonzentration 


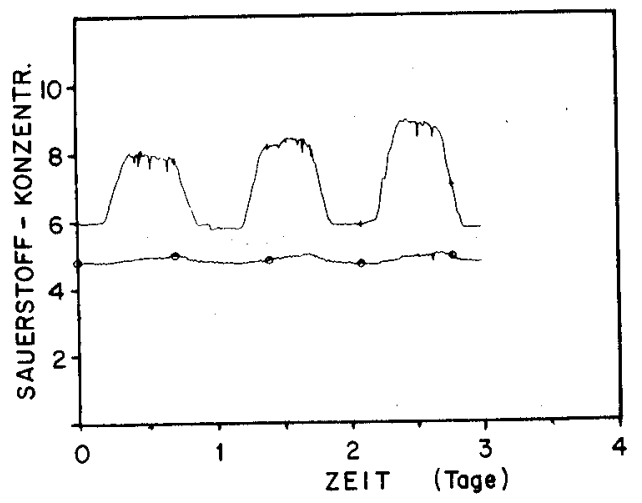

Abb.5. Messdaten der Rinne $(O=$ Sauerstoffkonzentration am Rinnenanfang, $+=$ für die Identifikation verwendete Sauerstoffkonzentration am Rinnenende).

Figure 5. Raw data from the artificial channel $(O=$ concentration of dissolved oxygen at the beginning of the channel, $+=$ concentration of dissolved oxygen at the end of the channel used for identification).

$\mathrm{O}_{\mathrm{gem}}(\mathrm{t})$ kompensiert. Die zeitliche Verschiebung zwischen $\mathrm{O}(\mathrm{t})$ und $\mathrm{O}_{\mathrm{gem}}(\mathrm{t})$ hängt vom Wert des identifizierten Parameters $\mathrm{k}$ ab. Je grösser $\mathrm{k}$ ist, um so rascher erfolgt der Anstieg von $\mathrm{O}(\mathfrak{t})$ und um so kleiner ist die Verschiebung zwischen $O(t)$ und $O_{\text {gem }}(t)$, jedoch nimmt dadurch die Amplitude von $O(t) z u$. Der identifizierte Wert für $\mathrm{k}$ stellt somit einen Kompromiss dar zwischen richtiger (= rascher) Anstiegszeit und dadurch bedingtem Fehler wegen zu grosser. Amplitude oder richtiger Amplitude und dadurch bedingtem Fehler wegen der verzögerten Anstiegszeit.

- Es ist bekannt, dass einzelne Modellparameter nicht echte Konstanten sind. So ist zum Beispiel die Photosyntheserate $a$ selbst wieder eine Funktion der Lichtintensität [12]; somit müsste $a$ um Mittag durch ein Minimum gehen und nachts konstant bleiben. Da solche Abhängigkeiten durch das Modell nicht beschrieben werden, müssten sie durch die Gradientenmethode - welche die Parameter kontinuierlich identifiziert - aufgezeigt werden. Wie aus Abb. 4 ersichtlich, wurden für alle Parameter täglich wiederkehrende Maxima und Minima gefunden. Insbesondere schwankt $a$ zwischen einem Minimum am Mittag und einem Maximum um Mitternacht. Die Minima von a scheinen wegen seiner Abhängigkeit von der Lichtintensität plausibel zu sein. Die Maxima um Mitternacht haben jedoch keine physikalische Bedeutung, da $a$ für $I(t)=0$ beliebige Werte annehmen kann. Man würde jedoch annehmen, $a$ bliebe konstant, solange $I(t)=0$ ist. Dies ist nicht der Fall, da die Identifikationsalgorithmen die Parameter der Differenzengleichung (3) identifizieren und nicht diejenigen der Differentialgleichung (1). a [Gleichung (1)] ist über

$$
a=\frac{b \cdot \ln (a)}{T_{s} \cdot(a-1)}
$$


mit dem identifizierten Parameter $b$ in Gleichung (3) verbunden. Wie aus Gleichung (17) hervorgeht, hängt $a$ noch vom Parameter a $a b$, der auch in der Nacht definiert ist und variieren kann.

Es ist jedoch zu beachten, dass alle identifizierten Parameter oft zwischen den täglichen Schwankungen noch weitere Minima und Maxima aufweisen. Da I $(t)$ nicht gemessen wurde, kann nicht entschieden werden, ob die Schwankungen der Parameter auf Modellfehler oder Änderungen in der Lichtintensität zurückzuführen sind.

Die Arbeit hat zu den folgenden Erkenntnissen geführt:

- Die in der Arbeit von Schurr und Ruchti vorgeschlagene Methode zur Bestimmung der Parameter des modifizierten Streeter-Phelps-Modells anhand der Sauerstoffdaten nur einer Messelektrode gründet auf zu einfachen Annahmen. Die Sauerstoffkonzentration muss an mindestens zwei örtlich getrennten Messorten gemessen werden. Diese Forderung ist gleichbedeutend mit einer Erweiterung [11] des mathematischen Modells:

$$
\frac{\delta \mathrm{O}(\mathrm{x}, \mathrm{t})}{\delta \mathrm{t}}=-\frac{\delta \mathrm{O}(\mathrm{x}, \mathrm{t})}{\delta \mathrm{x}} \cdot \mathrm{u}-\overleftarrow{\mathrm{k}} \mathrm{O}(\mathrm{x}, \mathrm{t})+\overrightarrow{\mathrm{k}} \mathrm{O}_{\mathrm{A}}+\alpha \mathrm{I}(\mathrm{x}, \mathrm{t})-\beta
$$

$\mathrm{u}=$ Fliessgeschwindigkeit des Flusses.

- Die Wahl der Standorte der Messelektroden ist von ausschlaggebender Bedeutung. Es muss gewährleistet sein, dass innerhalb des durch die beiden Messelektroden abgegrenzten Flussabschnittes Sauerstoffkonzentrationsänderungen nur durch die in der Gleichung (18) vorkommenden Terme hervorgerufen werden. Andere Möglichkeiten der Sauerstoffkonzentrationsänderungen, gegeben beispielsweise durch Stauwehre, in den Flussabschnitt einfliessende Abiwässer usw., müssen ausgeschlossen werden.

- Anstelle der durch die Gleichung (13) gegebenen Lichtintensität sollten die Werte $I(x, t)$ aufgrund echter Messwerte geschätzt werden.

- Für Untersuchungen mit einem erweiterten Modell dürfte die Gradientenmethode der Methode der Differentialapproximation vorzuziehen sein, da się allein die beobachteten und hier qualitativ bestätigten Veränderungen der Modellparameter nachweisen kann.

Die Kenntnis der biologischen Aktivitätsraten $\alpha$ und $\beta$ für einen Flussabschnitt erlaubt ein vertieftes Verständnis dessen Zustandes. Ein Modell zur Schätzung der Parameter $a$ und $\beta$ aus einfachen physikalischen und chemischen Grössen scheint deshalb wünschbar. Die vorliegende Arbeit zeigt, dass das Modell [Gleichungen (1) und (2)] auf zu einfachen Annahmen beruht und eine Erweiterung des Modells - zum Beispiel Gleichung (18) - notwendig ist. Die in der vorliegenden Arbeit verwendeten Methoden zur Verifikation eines Modells können grundsätzlich auch auf verfeinerte Modelle angewendet werden.

\section{Zusammenfassung}

Mit zwei Identifikationsalgorithmen (Differentialapproximation und Gradientenmethode) wurden die Modellparameter eines einfachen, nur zeitabhängigen Fluss- 
modells anhand von Daten der Aare (Bern und Brugg) und Daten einer künstlichen Rinne identifiziert. Die Differentialapproximation liefert für die Gesamtheit aller Sauerstoff- und Lichtintensitätswerte einen Wert für jeden zu identifizierenden Parameter. Im Gegensatz dazu erhält man mit der Gradientenmethode für je zwei aufeinanderfolgende Sauerstoff- und Lichtintensitätswerte je einen Wert für jeden zu identifizierenden Parameter.

Die Simulation führte bei der Differentialapproximation nur bei den Parametern zu anscheinend vernünftigen Ergebnissen, welche aus den Daten von Bern berechnet worden sind. Das offensichtliche Versagen des Modells für die Daten von Brugg kann damit erklärt werden, dass die Messung der Sauerstoffkonzentration unterhalb eines Stauwehrs erfolgt. Der vermutete Sauerstoffeintrag durch das Stauwehr verletzt die Modellannahme der Ortsunabhängigkeit der Sauerstoffkonzentration. Die unbrauchbaren Resultate für die ebenfalls in Funktion des Ortes stark ändernden Sauerstoffdaten der Rinne bestätigen diese Vermutung.

Die Simulation der Sauerstoffkonzentration mit den durch die Gradientenmethode gefundenen Parametersätzen ergibt erwartungsgemäss für alle Messorte ausgezeichnete Resultate. Jedoch konnte für keinen der drei Messorte eine eindeutige Erklärung für die zum Teil unregelmässigen Schwankungen der Parameter in Funktion der Zeit gefunden werden, da die Lichtintensität nicht gemessen, sondern nur simuliert wurde.

Die wichtigsten Resultate der Arbeit sind:

1. Das einfache, nur zeitabhängige Modell ist zur Beschreibung der Dynamik der Sauerstoffkonzentration in einem Fluss ungeeignet. Eine Erweiterung auf ein orts- und zeitabhängiges Modell ist notwendig.

2. Die Standortwahl der Sauerstoffmeßsonden ist von ausschlaggebender Bedeutung für die Anwendbarkeit des Modells.

3. Die Messung und modellgerechte Interpretation der Werte für die Lichtintensität bedarf weiterer Abklärungen.

\section{SUMMARY}

With two identification algorithms (the differential approximation and the gradient method) the parameters of a simple, time dependent model of a river were identified, using the data of the river Aare (stations Bern and Brugg) and the data of an artificial channel. The differential approximation gives, for the whole set of data for oxygen and light intensity, one value for each parameter to be identified. With the gradient method however, one obtains for every consecutive pair of values for oxygen and light intensity, one value for each parameter to be identified.

By the simulation with the differential approximation apparently good results are obtained only for those parameters calculated from the data of station Bern. The obvious failure of the model for the data of station Brugg can be explained by the fact that the measurements of the oxygen concentration were made below a weir. The assumed intake of oxygen due to the weir violates the assumption in the model, that the oxygen concentration is not a function of the location. The unusable results for the oxygen data obtained from the artificial channel, where the oxygen also varies with the location, verifies this assumption.

The simulation of the oxygen concentration with the sets of parameters obtained by the gradient method gives of course very good results. However it was not possible to give an explanation for any of the three locations with respect to the variation in time of the parameters since the light intensity was not measured but only simulated. 
The most important results of this work are: 1 . The simple, time dependent model is not adequate to describe the dynamics of oxygen concentration in a river. An extension to a time-space dependent model is necessary. 2. The applicability of the model depends heavily on the place where the oxygen measurements were taken. 3. The measurement and adequate interpretation of the light intensity values obtained must be investigated in a separate work.

\section{RESUME}

A l'aide de deux algorithmes d'identification (approximation différentielle et méthode du gradient) les paramètres d'un simple modèle fluvial ne dépendant que de variations temporelles furent identifiés en se basant sur des données de l'Aar (stations Berne et Brugg) et sur des données d'une rigole. L'approximation différentielle fournit pour l'ensemble de toutes les données d'oxygène et d'intensité lumineuse une valeur pour chaque paramètre à identifier. Avec la méthode du gradient cependant on obtient pour chaque paire consécutive de données d'oxygène et d'intensité lumineuse une valeur pour chaque paramètre à identifier.

Lors de la simulation avec l'approximation différentielle, on n'obtient apparemment de bons résultats que pour les paramètres qui furent basés sur les données de la station de Berne. L'inapplicabilité évidente du modèle aux données provenant de la station de Brugg est due au fait que la concentration d'oxygène fut mesurée en dessous d'une digue-réservoir. L'apport d'oxygène probable fourni par la digue ne respecte pas l'hypothèse du modèle selon laquelle la concentration d'oxygène n'est pas soumise aux variations locales. Les résultats inutilisables pour les données d'oxygène de la rigole, où la concentration d'oxygène varie également en fonction du lieu, confirment cette hypothèse.

La simulation de la concentration d'oxygène avec les groupes de paramètres déterminés par la méthode du gradient donne évidemment de très bons résultats. Toutefois, il ne fut pas possible d'expliquer les variations temporelles des paramètres pour aucun des trois points de mesure vu que l'intensité lumineuse fut seulement simulée et non pas mesurée.

Les résultats les plus importants de ce travail sont: 1. Le modèle simple mais soumis seulement à des variations temporelles n'est pas approprié pour décrire la dynamique de concentration d'oxygène dans une rivière. Une extension à un modèle soumis à des variations locales et temporelles est nécessaire. 2. L'applicabilité du modèle dépend en grande partie du lieu où les mesures d'oxygène sont effectuées. 3. Les mesures et l'interprétation adéquate des valeurs d'intensité lumineuse obtenues requièrent une étude plus approfondie.

\section{LITERATURVERZEICHNIS}

1 Streeter, H.W., und Phelps, E. B.: A Study of the Pollution and Natural Purification of the Ohio River. III. Factors Concerned in the Phenomena of Oxidation and Reaeration. Publ. Health Bull. No, 146, US Publ. Health Service Washington, D.C., 1925.

2 Tsivoglou, E.C.: Tracer Measurement of Stream Reaeration. Federal Water Pollution Control Administration. US Department of the Interior. Washington, D.C., June 1967.

3 Longtin, J.R.: Radiological Safety. In: Symposium on Direct Tracer Measurement of the Reaeration Capacity of Streams, and Estuaries, July 7-8, 1970; co-sponsors - Georgia Institute of Technology and US Environmental Protection Agency, Water Pollution Control Research Series 16050 for $01 / 72$, p. 19-20.

4 O'Connor, D.J., und Dobbins, W.E.: Mechanism of Reaeration in Natural Streams. Trans. ASCE 123, 641 (1956).

5 Dobbins, W.E.: The Nature of Oxigen Transfer Coefficient in Aeration Systems. Part 2-1 of Biological Treatment of Sewage and Industrial Wastes. Hrsg. McCabe und Eckenfelder. Rheinhold, New York, N.Y., 1956.

6 Churchill, M.A., Elmore, H.L., und Buckingham, R.A.: Prediction of Stream Reaeration Rates. J. sanit. Eng. Div., Proc. ASCE 88, SA4, 1 (1962).

7 Koivo, H.N., und Koivo, A.J.: Optimal Estimation of Polluted Stream Variables. 3rd IFAC Symposium on Identification and System Parameter Estimation, 1973 (North-Holland - American Elsevier). 
8 Schurr, J.M., und Ruchti, J.: Kinetics of Oxigen Exchange, Photosynthesis, and Respiration in Rivers Determined from Time-Delayed Correlations between Sunlight and Dissolved Oxygen. Schweiz. Z. Hydrol. 37, 144 (1975).

9 Eykhoff. P.: System Identification, 303ff. John Wiley and Sons, London 1974.

10 Maletinsky. V., und Schaufelberger, W.: Suboptimum Adaptive Control. Proceedings of the 4th IFAC/IFIP International Conference on Digital Computer Applications to Process Control. Lecture Notes in Economics and Mathematical Systems, B. 93, 129ff. Springer-Verlag, Berlin 1974.

11 Wetzel, J.C.: Dissertation, in Vorbereitung.

12 Eichenberger, E., und Wuhrmann, K.: Growth and Photosynthesis during the Formation of a Benthic Algal Community. Verh. int. Ver. Limnol. 19, 2035 (1975).

Adresse der Autoren: Dr. P.E. Erni und Dr. J. Ruchti, EAWAG, CH-8600 Dübendorf.

\section{Anhang:}

\section{Herleitung der Differenzengleichung (3) aus den Gleichungen (1) und (2)}

Durch Einsetzen von Gleichung (2) in die Differentialgleichung (1) erhält man

$$
\dot{O}(t)=a^{\prime} \cdot O(t)+b^{\prime} \cdot I(t)+c^{\prime}
$$

mit

$$
\begin{aligned}
& \mathrm{a}^{\prime}=-\overleftarrow{\mathrm{k}} \\
& \mathrm{b}^{\prime}=a \\
& \mathrm{c}^{\prime}=\overrightarrow{\mathrm{k}} \mathrm{O}_{\mathrm{A}}-\beta .
\end{aligned}
$$

Mit einer Variablentransformation

$$
\begin{aligned}
& \mathrm{z}(\mathrm{t})=\mathrm{a}^{\prime} \cdot \mathrm{O}(\mathrm{t})+\mathrm{c}^{\prime}, \\
& \dot{z}(\mathrm{t})=\mathrm{a}^{\prime} \cdot \dot{O}(\mathrm{t})
\end{aligned}
$$

zur Elimination des konstanten Gliedes c' erhält man

$$
\dot{z}(t)=a^{\prime} \cdot z(t)+a^{\prime} \cdot b^{\prime} \cdot I(t)
$$

Integration von Gleichung (A 7) mit Hilfe der Laplace-Transformation:

$$
p \cdot z(p)-z(0)=a^{\prime} \cdot z(p)+a^{\prime} \cdot \dot{b}^{\prime} \cdot I(p)
$$

Aufgelöst nach $\mathrm{z}(\mathrm{p})$ und $\mathrm{mit} I(\mathrm{p})=1 / \mathrm{p}$ erhält man

$$
z(p)=\frac{z(0)}{p-a^{\prime}}+\frac{a^{\prime} \cdot b^{\prime}}{p\left(p-a^{\prime}\right)} .
$$

Rücktransformation in den Zeitbereich:

$$
z(t)=e^{a^{\prime} \cdot t} \cdot z(0)+b^{\prime}\left(e^{a^{\prime} \cdot t}-1\right) \cdot I(t) .
$$


Umwandlung von Gleichung (A10) in eine Differenzengleichung:

$$
z(k+1)=e^{a^{\prime} \cdot T_{s}} \cdot z(k)+b^{\prime}\left(e^{a^{\prime} \cdot T_{s}}-1\right) \cdot I(k)
$$

Variablenrücktransformation $(\mathrm{z} \rightarrow \mathrm{O})$

$$
\begin{aligned}
& \mathrm{z}(\mathrm{k}+1)=\mathrm{a}^{\prime} \cdot \mathrm{O}(\mathrm{k}+1)+\mathrm{c}^{\prime}, \\
& \mathrm{z}(\mathrm{k})=\mathrm{a}^{\prime} \cdot \mathrm{O}(\mathrm{k})+\mathrm{c}^{\prime} .
\end{aligned}
$$

Eingesetzt in Gleichung (A11) ergibt

$$
O(k+1)=e^{a^{\prime}} \cdot T_{s} \cdot O(k)+\frac{b^{\prime}}{a^{\prime}} \cdot\left(e^{a^{\prime}} \cdot T_{s}-1\right) \cdot I(k)+\frac{c^{\prime}}{a^{\prime}}\left(e^{a^{\prime}} \cdot T_{s}-1\right) .
$$

Werden in die Differenzengleichung (A14) die Gleichungen (A2)-(A4) eingesetzt, so erhält man die gesuchte Differenzengleichung (3). 